\title{
High power microwave switch employing electron beam triggering with application to active rf pulse compressors
}

\author{
O. A. Ivanov, ${ }^{1,2}$ V. A. Isaev, ${ }^{1}$ M. A. Lobaev, ${ }^{1}$ A. L. Vikharev, ${ }^{1,2}$ and J. L. Hirshfield ${ }^{2,3}$ \\ ${ }^{1}$ Institute of Applied Physics RAS, Nizhny Novgorod, 603600 Russia \\ ${ }^{2}$ Omega-P, Inc., New Haven, Connecticut 06510, USA \\ ${ }^{3}$ Department of Physics, Yale University, New Haven, Connecticut 06511, USA
}

(Received 14 April 2011; published 17 June 2011)

\begin{abstract}
Switch for modulation of the $Q$ factor of a multimode rf energy storage resonator is described. The $\mathrm{rf}$ energy is stored in a cylindrical resonator in the $\mathrm{TE}_{02}$ mode, and switched out from it in the $\mathrm{TE}_{01}$ mode. The operating principle of the switch is based on effecting a fast increase in the $\mathrm{TE}_{02} \rightarrow \mathrm{TE}_{01}$ mode coupling coefficient when an electron beam is injected into the switch cavity. This paper focuses on use of the switch in an active rf pulse compressor. Use of the electron beam allows one to separate the switching element from the compressor itself, and therefore to eliminate limitations to the level of switched power that exists for other switches. The pulse compressor was tested at low power at a frequency of 11.424 GHz. The level of peak power gain achieved in these experiments was 19-21, with compressed pulse widths of 30-40 ns. Good stability and repeatability of the shape of compressed pulses was observed. High power versions of this switch appear promising for use in active GW-level microwave compressors.
\end{abstract}

DOI: 10.1103/PhysRevSTAB.14.061301

PACS numbers: $84.40 . \mathrm{Az}, 84.90 .+\mathrm{a}$

\section{INTRODUCTION}

It is widely accepted that microwave pulse compression is required for future $\mathrm{TeV}$-scale electron-positron linear colliders using external $\mathrm{rf}$ drive in order to achieve the necessary high pulsed power levels. For this purpose, passive compression has been developed and implemented $[1,2]$, and active pulse compression has recently been investigated [3-5]. Passive compression relies upon phase modulation of the primary pulse; currently, the maximum power achieved by passive compression amounts to $500 \mathrm{MW}$ for a compressed pulse duration of $400 \mathrm{~ns}$ at $11.4 \mathrm{GHz}$ [2]. The drawbacks of passive compression systems include a relatively low power gain, unless operation at low efficiency can be tolerated. In [2], a power gain of about 3:1 was achieved at an efficiency of $60 \%$, using four klystrons with a total input power of $160 \mathrm{MW}$.

Active compressors in principle allow one to achieve higher power gains without diminution in efficiency, so that sources with lower powers can be used to achieve a given desired peak power, or greater peak power can be achieved for the same input power. Active compression is based on the storage of energy in a high- $Q$ microwave resonator or delay line followed by a rapid increase of the coupling between the resonator and the load ( $Q$ switching) using the active element, e.g., a fast switch. For energy storage, high- $Q$ multimode resonators are usually used,

Published by the American Physical Society under the terms of the Creative Commons Attribution 3.0 License. Further distribution of this work must maintain attribution to the author(s) and the published article's title, journal citation, and DOI. which operate in axisymmetric $\mathrm{TE}_{0 n}$ modes with low Ohmic losses and high electric strength. The main problem to be overcome when multimode systems are sought is the realization of a high-efficiency, electrically robust, fastacting ( $\leq 10 \mathrm{~ns}$ ) switch to rapidly change the resonator coupling to allow discharge of energy from the storage resonator to the load. Operation of switches for application at $\mathrm{X}$ band has been based on several distinctive approaches, including changes in the dielectric permittivity (i.e., rf conductivity) of semiconductors or ferroelectrics, caused by incident pulsed optical radiation [6,7] or by an external bias voltage [8], and generation of pulsed plasma in the switch [3-5]. The best experimental results [4,5] obtained so far have been for compressors employing plasma switches, though the parameters achieved are still far from those required for accelerator applications. Power levels are limited principally by the occurrence of multipactor discharges on the surface of dielectrics or semiconductors located within the switch, and with electric breakdown at or near the materials used in the switches. Indeed, most switch designs involve the somewhat contradictory requirements that the electric field on the surface of a dielectric element (such as a quartz tube in which a plasma discharge is to be ignited) should be sufficiently low to avoid spontaneous breakdown, but also sufficiently high to guarantee efficient switching when triggered breakdown is initiated.

The work described in this paper is based on a new switch employing electron beam triggering. Highefficiency energy output from the multimode resonator of the microwave compressor can be demonstrated for this type of switch. The switch is free of the drawbacks limiting 
the value of the switched power in other types of switches and has high electric strength, stability, and the possibility to vary the switching ratio within a wide range. These attributes arise mainly from the absence of insulators in the switch, and from its role to detune a cavity, rather than to tune it. The switch thus has properties that lend it to use in schemes of active pulse compression for future particle accelerators.

\section{GENERAL SCHEME OF PULSE COMPRESSOR WITH AN ELECTRON BEAM SWITCH BASED ON MODE CONVERSION}

In an rf pulse compressor using this switch, energy is accumulated in the $\mathrm{TE}_{02}$ mode (providing high $Q$ and electric strength) and extracted in $\mathrm{TE}_{01}$ mode, both in a cylindrical waveguide storage resonator. Operation of the compressor is based on a sharp increase in the $\mathrm{TE}_{02} \rightarrow \mathrm{TE}_{01}$ mode conversion coefficient during electron beam injection in the switch. Thus, the principle of operation of the switch is similar to the principle of operation of the plasma switch as described in [5,9], but with switching by an electron beam instead of plasma. The switch possesses resonant properties, which ensures a significant decrease in requirements for the quality of the electron beam. Application of an electron beam for changing frequency characteristics of a switch resonator has other advantages. In this case it is not necessary to install additional elements in the switch, a cold cathode may be used, and the cold cathode may be situated out of the switch resonator. The electric strength of the switch will be determined by the parameters of the empty resonator in vacuum and, hence, remove limitations to a significant extent due to gas breakdown or multipactor for the switched microwave power.

The design and the principle of operation of a microwave compressor with the proposed switch at $\mathrm{X}$ band are illustrated in Fig. 1. Radiation generated by an rf source in a $\mathrm{TE}_{10}$ mode rectangular waveguide is transformed by a mode converter into a $\mathrm{TE}_{01}{ }^{\mathrm{O}}$ mode and fed to the input of the compressor at a frequency of $11.424 \mathrm{GHz}$. The compressor consists of a coupling device (I) (input and output waveguides, in which the $\mathrm{TE}_{02}$ mode is cut off), input horn (II), a section of cylindrical waveguide (III), and switch (IV). The input horn, cylindrical waveguide, and switch form a storage cavity for the $\mathrm{TE}_{02}$ mode. The cylindrical waveguide (III) includes a section of variable length, which can be changed using a special mechanical drive so as to tune the cavity to a desired working frequency. In the regime of energy storage, a small fraction $(\sim 1 \%-3 \%)$ of the $\mathrm{TE}_{01}$ mode supplied from the rf source via the input waveguide is converted with the aid of the switch into the $\mathrm{TE}_{02}$ mode. This mode is completely reflected from the input horn (II). As a result, the microwave energy is stored in the cavity in the $\mathrm{TE}_{02}$ mode.

Let us consider the principal of operation of the switch with an electron beam, Figs. 1(b) and 1(c). It consists of the taper (1), in which the modes are converted $\left(\mathrm{TE}_{02} \rightarrow \mathrm{TE}_{01}\right.$ and $\mathrm{TE}_{01} \rightarrow \mathrm{TE}_{02}$ ), and the section of circular waveguide

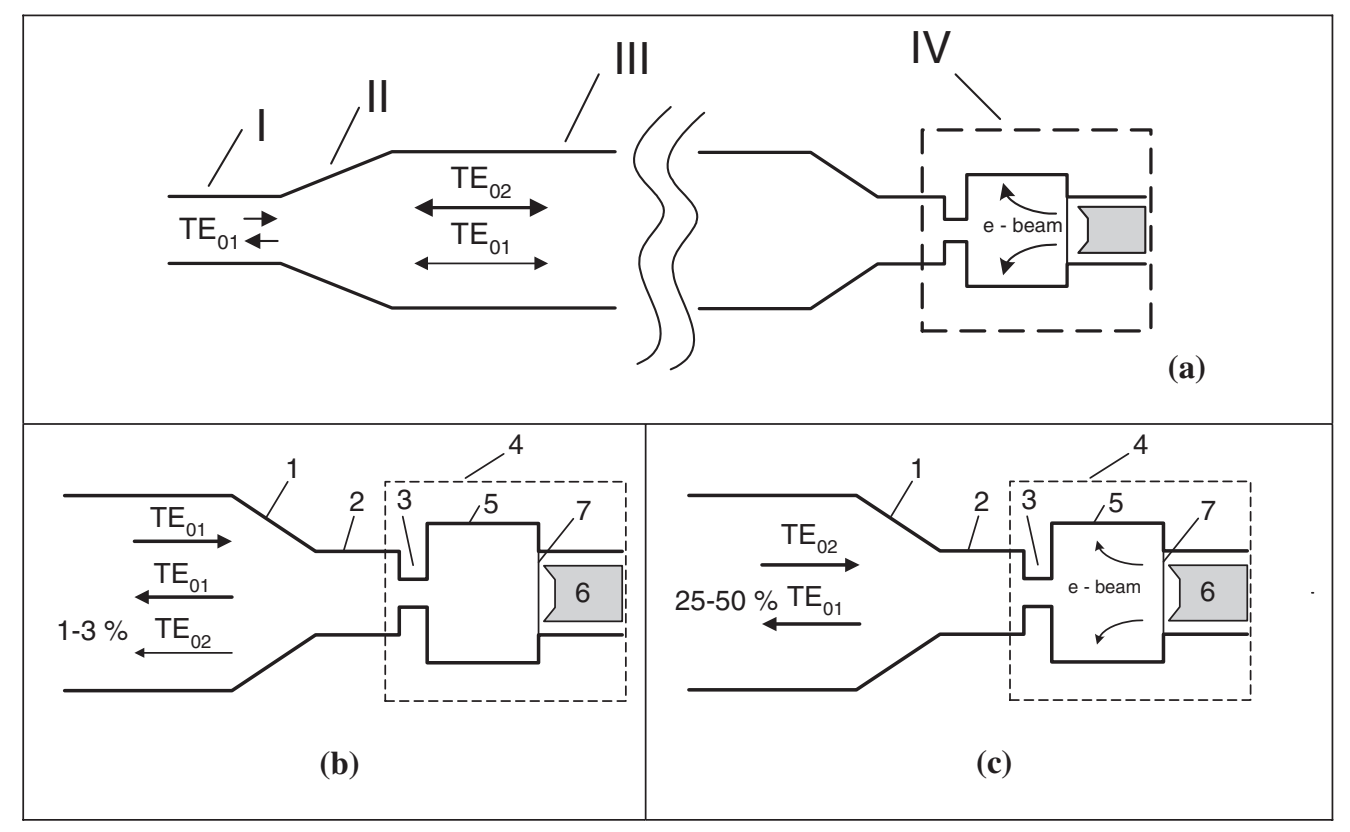

FIG. 1. (a) Schematic diagram of a pulse compressor with the switch employing an electron beam, illustrating switch operation in the energy storage (b) and pulse extraction regimes (c): I—input $\mathrm{TE}_{01}$ mode waveguide, II—input horn, III—cylindrical waveguide cavity, IV—electron beam switch; 1-taper, 2-circular waveguide, 3-diaphragm, 4-resonator, 5-stepped widening, 6-cathode, and 7-anode (special diaphragm). 
(2) which ensures the phase changes required for mutual interference of the $\mathrm{TE}_{01}$ mode power reflected from the taper (1) and the resonator (4). Thus, in the regime of energy storage only a small fraction of the $\mathrm{TE}_{01}$ mode supplied from the rf source is converted into the $\mathrm{TE}_{02}$ mode, as in Fig. 1(b). The switch resonator is formed by iris diaphragm (3), a section of a cylindrical waveguide (5), and rear wall (7) (special diaphragm) that also acts as the anode. The length of the resonator could be changed externally using an adjustment device. When the electron beam is injected into the switch resonator, the phase of the signal reflected from the resonator (4) changes, the $\mathrm{TE}_{01}$ mode reflected from the taper (1) and from the cavity sum up in phase, and the $\mathrm{TE}_{02} \rightarrow \mathrm{TE}_{01}$ conversion ratio rises up to $25 \%-50 \%$, as in Fig. 1(c).

The switch resonator shifts the phase of the wave reflected from it depending on whether the resonator is in, or out, of resonance. The reflection coefficient from the resonator is determined by the following expression [10]:

$$
R=1-\frac{2 \beta_{r}}{\left(1+\beta_{r}\right)\left(1+2 i Q_{L R} \frac{\omega-\omega_{0}}{\omega_{0}}\right)}
$$

where $\beta_{r}=Q_{0} / Q_{e}, Q_{0}$ is the Ohmic $Q$ factor, $Q_{e}$ is the coupling $Q$ factor, $Q_{L R}=Q_{0} Q_{e} /\left(Q_{0}+Q_{e}\right)$ is the loaded $Q$ factor of the switch resonator, $\omega_{0}$ is the resonance frequency of the resonator, and $\omega$ is the frequency of the incident wave. In this case, when $Q_{0} \gg Q_{e}$ the waves reflected from the resonator out of resonance and in resonance have the same amplitudes but opposing phases. The shape of the resonance curve and the frequency dependence of the oscillation phase are shown in Fig. 2.

It is seen from Fig. 2 that even a minor variation of the eigenfrequency of the cavity results in a sharp change in the phase of the signal reflected from the cavity. This feature of resonators may be used to develop a microwave switch. For example, if an in-resonance cavity is quickly driven out of resonance (change its resonance frequency),

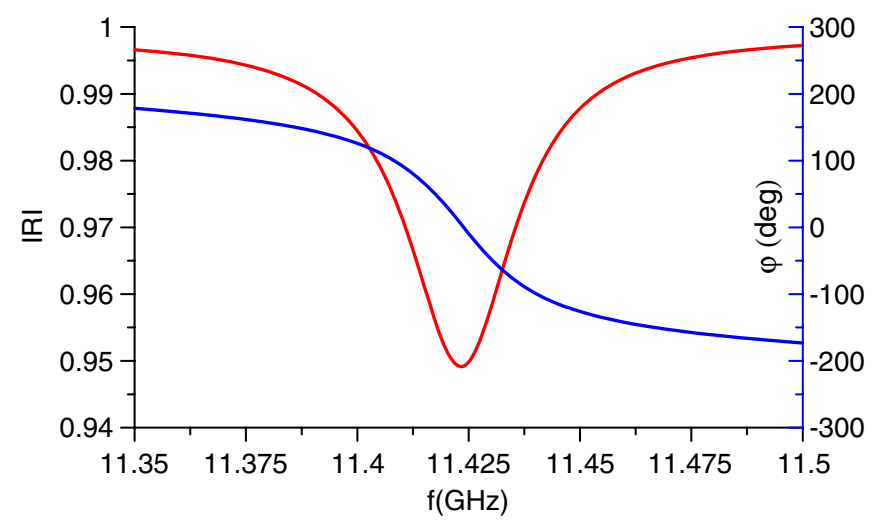

FIG. 2. Resonance characteristics of switch: resonance curve (red); dependence of the phase of the reflected signal on the frequency of the incident EM wave (blue).
TABLE I. Parameters of storage and switch resonators.

\begin{tabular}{lllcc}
\hline \hline$Q_{L R}$ & $\beta_{r}$ & $Q_{0}$ & $Q_{S}$ & $\beta$ \\
\hline 554 & 54 & $5.4 \times 10^{4}$ & $1.6 \times 10^{4}$ & 2.4 \\
380 & 76.5 & $6.1 \times 10^{4}$ & $1.7 \times 10^{4}$ & 2.6 \\
224 & 127 & $7 \times 10^{4}$ & $1.5 \times 10^{4}$ & 3.5 \\
\hline \hline
\end{tabular}

then according to Eq. (1) it will lead to shifting of the phase of the wave reflected from it by $\Delta \varphi \approx \pi$. The time of phase changing, $\tau_{p}$, will be determined by the time of oscillation stabilization in the resonator, i.e., $\tau_{p}=$ $Q_{L R} / \omega_{0}$. For example, when the resonator $Q$ factor is $Q_{L R}=300$ and the frequency is $f_{0}=11.4 \mathrm{GHz}$, the characteristic time of phase shifting will be $\tau_{p} \approx 5 \mathrm{~ns}$.

It should be noted that achievement of the maximum power of compressed pulses requires a high value of the $Q$ factor of the storing resonator in the compressor. Therefore, a switch with an electron beam should not produce significant losses during energy storage in the compressor. To examine this requirement, we calculated losses for several types of switches based on cylindrical copper resonators excited in the $\mathrm{TE}_{01}$ mode. Calculations were performed using the finite-difference time-domain method and allowing for the $\mathrm{TE}_{02} \leftrightarrow \mathrm{TE}_{01}$ mode conversion coefficient, which determines the parameter $\beta$ of the storage resonator at the stage of energy storing in the compressor. The size of the switch cavity and the diameter of the input diaphragm were chosen based on the calculation results. The results of calculations for a length of storage resonator $L=100 \mathrm{~cm}$ and frequency of the incident wave $f 0=11.4 \mathrm{GHz}$ are shown in Table I.

Here $Q_{0}$ is the Ohmic $Q$ factor, and $Q_{S}$ is the loaded $Q$ factor of storage resonator. The calculations showed that the storing resonator $Q$ factor $Q_{S} \geq 10^{4}$ can be achieved, when the $Q$ factor of the switch resonator is $Q_{L R} \approx$ 200-500 and $\beta_{r} \geq 50$.

\section{DESIGN OF THE SWITCH AND ELECTRON BEAM PARAMETERS NECESSARY FOR SWITCHING}

Let us consider parameters of an electron beam required to change the phase of the electromagnetic wave reflected from a switch resonator. To do this, we will estimate the electron density $N_{e}$ and the total number of electrons $N_{\Sigma}$ required to drive the resonator off resonance (i.e., to shift the frequency to half width of the resonance curve, $\Delta f_{0.5}$ ) when the beam is injected into the resonator. The shift of resonance frequency $\Delta f$, caused by injection of electrons into the resonator, can be evaluated from perturbation theory using the following formula [11]:

$$
\frac{\Delta f}{f_{0}}=\frac{1}{2 N_{c}} \frac{\int_{V_{p l}} N_{e} E_{q}^{2} d V}{\int_{V_{r}} E_{q}^{2} d V},
$$


where $f_{0}$ is the inherent resonance frequency of the resonator, $N_{c}$ is the critical electron density (for $\left.f_{0}=11.4 \mathrm{GHz}, N_{c}=1.6 \times 10^{12} \mathrm{~cm}^{-3}\right), V_{r}$ and $V_{p l}$ are volumes of the resonator and the space occupied by the electrons, respectively, and $E_{q}$ is the intensity of the electric field of the excited resonator mode. Let us assume that the resonator for the phase shifter has a $Q$ factor $Q_{L}=300$ and a volume $V_{r} \approx 12 \mathrm{~cm}^{3}$. In this case, Eq. (2) yields the following results: (i) When the beam is injected into the volume of the maximum of the electric field, the total number of electrons in the resonator must exceed the value of $N_{\Sigma} \geq 2 N_{C} V_{r} A / Q_{L} \approx 6.6 \times 10^{10}$. Here $A$ is the form factor determined by the distribution of the field in the resonator (for the $\mathrm{TE}_{01}$ mode, $A \approx 0.5$ ). (ii) When the beam is injected uniformly into the volume of the resonator, the density of electrons in the resonator must exceed the value of $N_{e} \geq 2 N_{C} / Q_{L} \approx 1.07 \times 10^{10} \mathrm{~cm}^{-3}$, and the total number of electrons is $N_{\Sigma} \geq 1.3 \times 10^{11}$.

Note the parameters of the electron beam required to create this number of electrons. The value of the electron current may be determined from the following expressions:

$$
I[A]=e N_{c} S V_{e}=e N_{\Sigma} V_{e} / L,
$$

where $e$ is the electron charge, $S$ and $L$ are the area of the transverse cross section and the length of the electron beam, respectively, $V_{e}=5.92 \times 10^{7} \cdot \sqrt{U}$ is the velocity of electrons, and $U$ is the voltage at the electron beam. The calculation showed that the beam current must be no lower than $250 \mathrm{~A}$ for $N_{\Sigma} \geq 10^{11}$ at $U \sim 100 \mathrm{kV}$ and $L \sim 1 \mathrm{~cm}$. The possibility of changing the phase of the wave reflected from the cavity by using an electron beam was demonstrated in $[12,13]$ for the millimeter and centimeter wavelength bands.

Evidently, successful operation of the switch is largely dependent on the shape and mutual arrangement of the anode and the cathode. For example, in [13] was described the switch with $T$-shaped stainless-steel blade cathode that

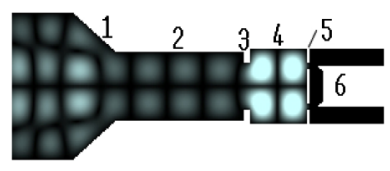

(a)

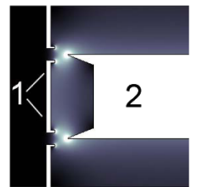

(b)

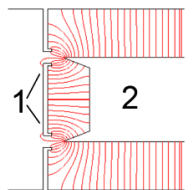

(c)

FIG. 4. Instantaneous distribution of the amplitude of the electric field (a) in the switch: 1-horn, 2-cylindrical waveguide, 3-diaphragm, 4-switch resonator, 5-cooper anode with slit, and 6-cathode. The distribution of the module of static electric field (b) and electric flux (c) in the switch: 1 -anode, 2-cathode.

was situated inside the switch resonator. The design of the switch allows various modifications provided the cathode is situated near the antinode of the field of the standing wave in the switch cavity. To ensure this, based on the calculations, we select such a design of the anode, cathode, and the switch resonator (Fig. 1) to achieve the maximum of the static field on the cathode situated outside the switch cavity and reaching values at which high field emission of electrons is possible. Photos of the cathode and anode parts of the electron beam switch are shown in Fig. 3. The electron beam was produced by a cylindrical cathode with edges having a radius of curvature of $0.2-0.3 \mathrm{~mm}$, as seen in Fig. 3(a). It is known that the field is enhanced at the sharp edges of the cathode, and under a sufficiently high voltage leads to an onset of electron field emission. The anode was made of a copper disk with a thickness of $2 \mathrm{~mm}$. A 2.8-mm wide circular slit was cut through the disk at a distance of $10 \mathrm{~mm}$ from its center. The central part of the anode was connected with main body (1) by four $0.5 \mathrm{~mm}$ thick legs (3) situated within the slit, Fig. 3(b). The center of the slit corresponded to the position of the field maximum in the $\mathrm{TE}_{01}$ mode and was situated directly opposite to the sharp edge of the cathode, as shown in Fig. 4(a).

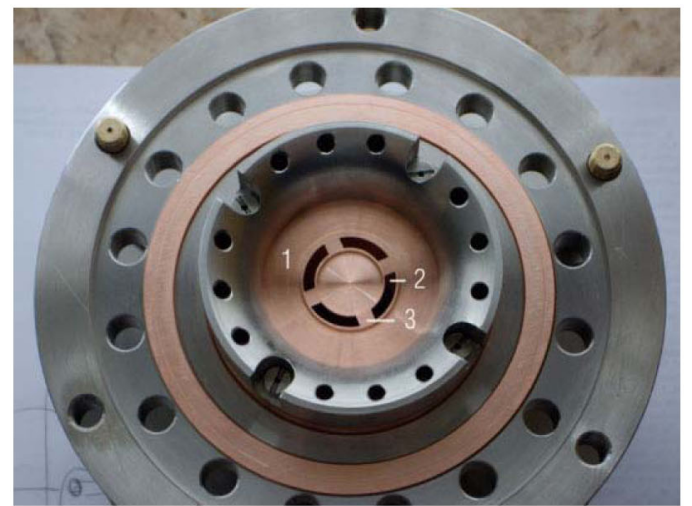

(a)

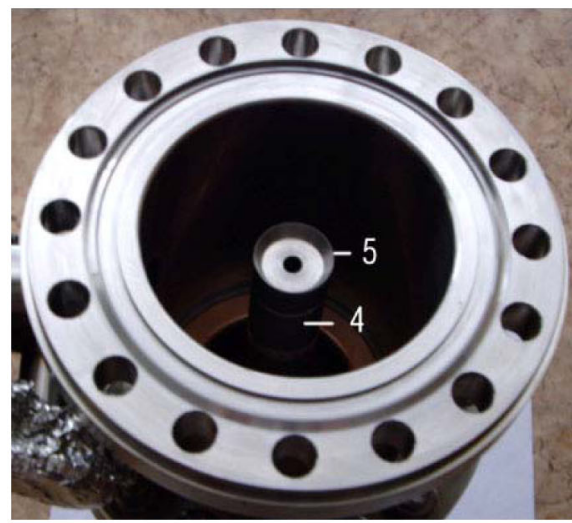

(b)

FIG. 3. Photo of the cathode (a) and anode (b) parts of the switch: 1—anode disk, 2—slit, 3-leg, 4-cathode holder, and 5 - cathode edge. 
The front wall of the anode acted simultaneously as a wall of the switch resonator. The width and depth of the slit (2) were chosen based on results of calculation so as to prevent the electromagnetic field from penetrating into the slit and leaking through slit (2). In this case, the anode plate with the slit reflects electromagnetic waves as a solid copper wall, and the presence of the slit does not degrade the intrinsic $Q$ factor of the switch resonator. Moreover, for the selected dimensions of the slit, the field of the $\mathrm{TE}_{01}$ mode excited in the resonator is not enhanced near the legs (3). As a result, the $Q$ factor of the switch remains high. Instantaneous distribution of the amplitude of the electric field in the switch is shown in Fig. 4(a). It is seen that the anode with slits introduces no discernible distortion to the structure of the electric field in the resonator. At the same time, the electron beam and the associated near-cathode plasma can penetrate freely into the cavity through slits in the anode.

The structure of the electrostatic field in the switch when a high-voltage pulse is fed to the cathode has been calculated. The calculation was performed for a cathode voltage $U=100 \mathrm{kV}$. The distribution of the module of static electric field and electric flux in the switch is shown in Figs. 4(b) and 4(c). The calculation shows that the intensity of the electric field in the vicinity of the sharp end (curvature of $0.3 \mathrm{~mm}$ ) reaches its maximum value of $E \sim 250 \mathrm{MV} / \mathrm{m}$, sufficient to achieve copious field emission from the cathode. Typically, for initiation of field electron emission, the value of the electric field at the cathode should exceed $\sim 100 \mathrm{MV} / \mathrm{m}$.

The switch to be discussed below used cathodes made of graphite or stainless steel and had sharp edges, as in Fig. 4. Parameters of the electron beam for such cathodes have been studied. To achieve this, pulses with an amplitude of $100 \mathrm{kV}$ and duration of $100 \mathrm{~ns}$ generated by a high-voltage pulser (HVP) were fed to the cathode placed near the switch cavity (as in Fig. 1). Figure 5(a) shows photos of the cathode taken at the instant a high-voltage pulse was applied to it. Cathode spots arising at the sharp edges of the cathode are distinctly seen. These spots are interpreted to be plasma puffs resulting from the explosion of microtips on the cathode due to field emission. These puffs are the primary sources of electrons in the beam.

The beam current was measured using a graphite collector installed at an antinode of standing wave in the switch resonator. The switch was evacuated with an ion pump to a pressure of $10^{-6}$ Torr. The electrons emitted from the cathode are accelerated in the gap between the anode and the cathode, pass through the slits in the anode and then are gathered by the collector. The collector current is measured using a noninductive resistor. A characteristic oscillogram of the collector current is shown in Fig. 5(b). Measurements showed that the beam current exceeded $250 \mathrm{~A}$ and had a duration $\sim 40 \mathrm{~ns}$. Thus, the HVP used earlier for the X-band plasma switch [5] can be used to excite a switch using an electron beam.

A prototype of this switch with an electron beam as in Fig. 5 should in principal be capable of engineering to survive several million rf pulses or more with good reliability, in order to confirm its rf properties. Switch lifetime depends on lifetime of the cathode, which in this instance consists of a sharp metal edge that could be eroded. To eliminate this effect, the cathode could be made of a material resistant to erosion, such as graphite or stainless steel, and the sharp corners rounded. For example, when equipped with a graphite cathode, a device with the same shape of cathode has worked for $100 \times 10^{6}$ shots at a current level of $5 \mathrm{kA}$, voltage of $500 \mathrm{kV}$, and a repetition rate ranging from 100 to $150 \mathrm{~Hz}$ [14]. Following this, no significant erosion of the cathode or anode was observed. In our case the required parameters of the electron beam are considerably lower $(I<1 \mathrm{kA}, U<100 \mathrm{kV}$,

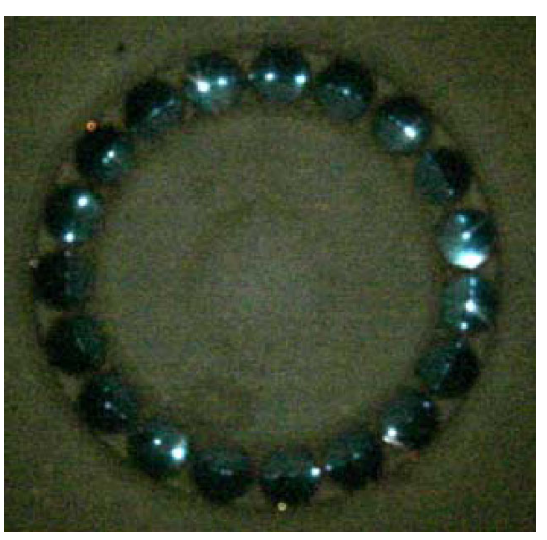

(a)

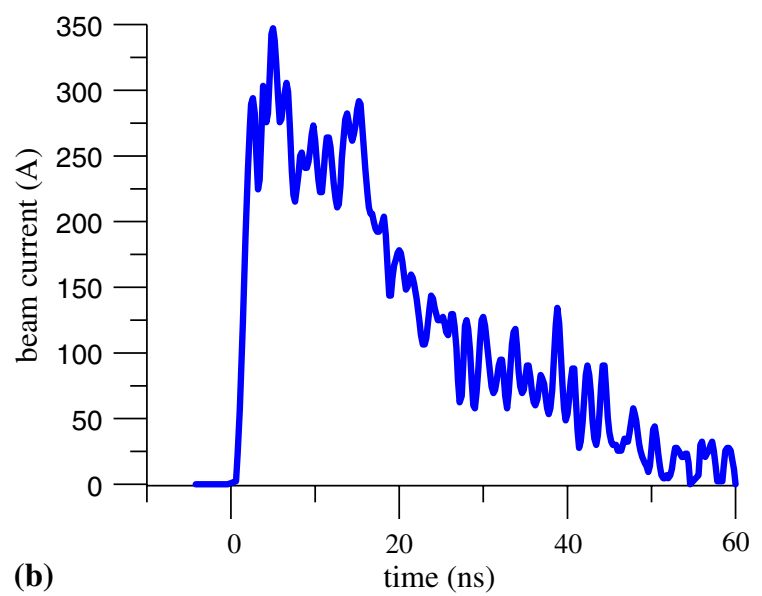

(b)

FIG. 5. (a) Cathode spots (photo made through the anode slots) at the instant of high-voltage pulse application $(U=100 \mathrm{kV}$, pulse duration $\tau=100 \mathrm{~ns}$ ) and oscillogram of the cathode current. 


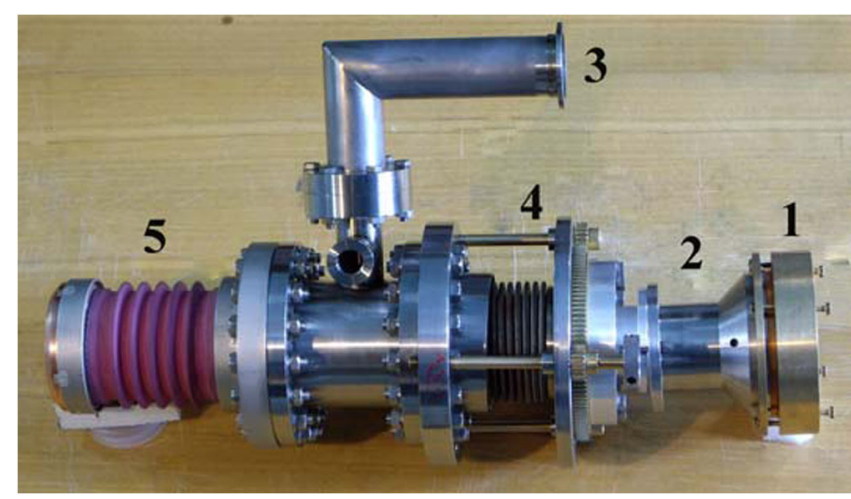

FIG. 6. Photo of the switch employing electron beam triggering: 1-taper, 2-cylindrical waveguide, 3-pumping port, 4-external tuning mechanism, and 5-insulator.

$f=100 \mathrm{~Hz}$ ). Therefore the erosion effect of the cathode should not pose a limitation to carrying out a proof-ofprinciple experiment.

\section{LOW POWER TEST OF X-BAND COMPRESSOR WITH ELECTRON BEAM SWITCH}

The operation of a microwave pulse compressor with the switch employing electron beam triggering was experimentally studied at a low rf power level. The general view of electron beam switch is shown in Fig. 6.

The storage resonator used in the experiments was made of duralumin and filled with air at atmospheric pressure. The compressor was excited using a low power microwave oscillator operating in a cw mode at $11.424 \mathrm{GHz}$. The scheme of the experiment and the general view of the experimental setup are shown in Fig. 7.

The radiation generated by a microwave oscillator in the form of a $\mathrm{TE}_{10}$ square-waveguide mode is transformed by mode converters (4) into a $\mathrm{TE}^{\mathrm{O}}{ }_{01}$ mode and fed to the input of a compressor at a frequency of $11.424 \mathrm{GHz}$. The compressor consists of input-output taper (6), a 120-cm-long section of cylindrical aluminum waveguide (7) with a diameter of $8 \mathrm{~cm}$, and electron beam switch $(8,15-20)$. The input horn, cylindrical waveguide, and switch form a storage cavity for $\mathrm{TE}_{02}$ mode. The cylindrical waveguide includes a section of variable length, which can be changed using a special mechanical drive so as to tune the cavity to a desired working frequency.

The frequency characteristic of the compressor was measured using a scalar network analyzer model $8757 \mathrm{C}$. The loaded $Q$ factor of the storage resonator, as measured experimentally, was $Q_{L}=(1.5-2.6) \times 10^{4}$, depending on the parameters of the switch resonator and on the length of the cylindrical waveguide (2), Fig. 1. The measured coupling coefficient (parameter $\beta$ ) of storage resonator was $\beta \sim 1$ and was optimized for $\mathrm{cw}$ excitation [3].

The compressor was switched from the regime of microwave power storage into the regime of power output by firing the HVP. A high-voltage pulse with amplitude of $100 \mathrm{kV}$ and duration of $100 \mathrm{~ns}$ was fed to the cathode of the switch. The distance between the anode and the stainlesssteel cathode was varied in the range $D_{a^{-c}}=1.5-5 \mathrm{~mm}$. The diameter of the cathode was $20 \mathrm{~mm}$, and the cathode had a sharp edge with a thickness of $0.2 \mathrm{~mm}$. The switch was evacuated with an ion pump to a pressure of $10^{-6}$ Torr. Electron injection resulted in a change in the

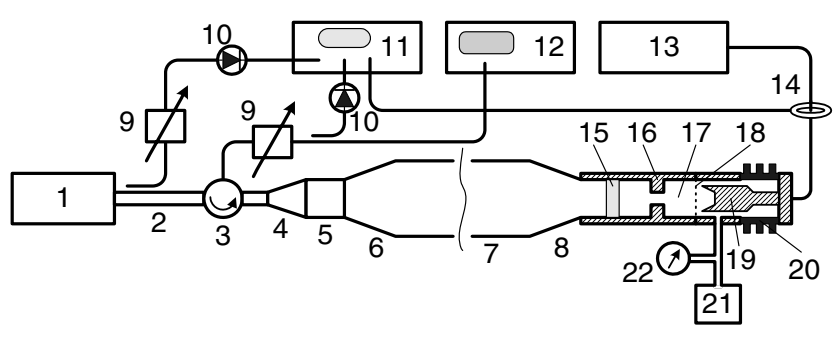

(a)

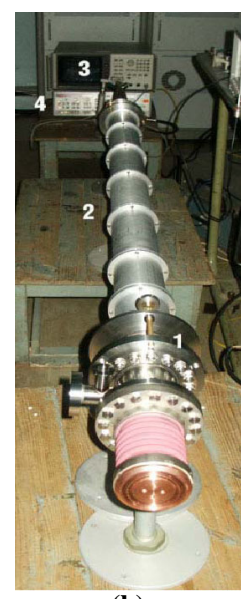

(b)

FIG. 7. (a) Schematic layout of the experiment: 1 -microwave generator, 2 -directional coupler, $3-$ circulator, $4-\mathrm{TE}_{10} \rightarrow \mathrm{TE} \mathrm{O}_{01} \mathrm{O}$ mode converter, 5-cylindrical waveguide, 6-input-output taper, 7-storage resonator, 8-taper, 9-attenuator, 10-microwave detector, 11-oscilloscope, 12-scalar network analyzer, 13-HV pulser, 14-Rogovsky coil; 15-microwave window, 16-movable diaphragm, 17—switch resonator, 18—anode (aluminum foil), 19-cathode, 20—insulator, 21—pumping port, and 22-vacuum gauge. (b) Photograph of the experimental setup: 1-electron beam switch, 2-storage resonator, 3-scalar network analyzer, and 4-microwave generator. 


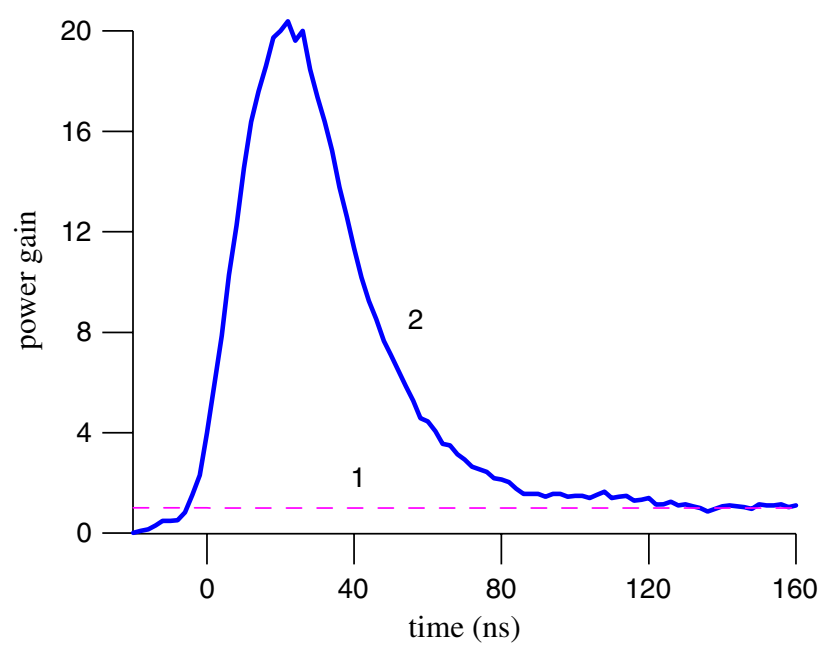

FIG. 8. Typical oscilloscope traces of the incident (1) and compressed (2) pulses for the switch employing electron beam triggering. The gap between anode and cathode is equal to $2.4 \mathrm{~mm}$.

resonance frequency of the switch resonator, loss of resonance, and, correspondingly, to a variation in the phase of the signal reflected from the resonator, as in Fig. 2. At the same time, the $\mathrm{TE}_{02} \rightarrow \mathrm{TE}_{01}$ transformation coefficient increased up to $30 \%-50 \%$. As a result, microwave energy stored in the resonator in the $\mathrm{TE}_{02}$ mode was emitted through the input horn in the $\mathrm{TE}_{01}$ mode, with a characteristic switching time of 5-6 ns. Figure 8 shows a typical oscillogram of compressed pulses obtained with the electron beam switch. The level of power gain achieved in these experiments was $K=19-21$, with a compressed pulse duration of 30-40 ns FWHM. The efficiency of energy extraction in the performed experiments was determined as the ratio of the energy in the compressed pulse to the energy stored in the resonator and reached $94 \%$. We also note the highly stabile appearance of the compressed output pulses.

It should be noted that for a short $(\sim 1.2 \mathrm{~m})$ storage resonator used in experiment the round-trip time along the resonator was equal to $\tau_{r} \sim 10 \mathrm{~ns}$ and was compared with switching time. Therefore at a low $\mathrm{TE}_{02} \rightarrow \mathrm{TE}_{01}$ transformation coefficient $(\sim 40 \%)$, the energy was extracted from the resonator during a time $t \sim(3-4) \tau_{r}$ and a compressed pulse was not of a rectangular shape and had exponentially reducing back front. The output pulse with rectangular profile can be obtained by using an electron beam switch with a higher transformation coefficient and a long storage resonator, for instance, with a resonance delay line of SLED-II type.

These test results obtained at low power level confirmed the operation of an electron beam switch of a new type for a $Q$-switched storage cavity of a microwave compressor which operates using the axisymmetric $\mathrm{TE}_{02} \leftrightarrow \mathrm{TE}_{01}$ cylindrical waveguide modes. This switch appears to have good prospects for use in active high power microwave compressors. Note also that tentative numerical simulations of operation of the compressor with this switch show that compression parameters can be improved by using a storage resonator made of copper and having a higher $Q$ factor.

\section{CONCLUSIONS}

As it has been already noted, the proposed switch does not have drawbacks which are characteristic of the switches of other types. It has significantly higher electric breakdown threshold, small dimensions, and high stability of operation (which was demonstrated in low power test). It is expected that the use of the switch as a component of an active microwave compressor will make it possible to use input pulses having a power of tens (possibly, hundreds) of megawatts and, correspondingly, produce compressed pulses at a gigawatt power level. Unlike a plasma switch, it is more resistant to heat loads, and therefore can be used at pulse repetition rates above $100 \mathrm{~Hz}$. Besides, such a switch is easily scalable and can operate, for example, at 12 and $34 \mathrm{GHz}$.

\section{ACKNOWLEDGMENTS}

This work was supported by the U.S. Department of Energy, Office of High Energy Physics, and in part by the Russian Foundation for Basic Research, Project No. 10-0800260.

[1] Z. Farkas I, IEEE-MTT-34, MTT-34, 1036, 1986.

[2] S. G. Tantawi, C. D. Nantista, V. A. Dolgashev, C. Pearson, J. Nelson, K. Jobe, J. Chan, K. Fant, J. Frisch, and D. Atkinson, Phys. Rev. ST Accel. Beams 8, 042002 (2005).

[3] R. A. Alvarez, D. P. Byrne, and R. M. Johnson, Rev. Sci. Instrum. 57, 2475 (1986).

[4] A. L. Vikharev, A. M. Gorbachev, O. A. Ivanov, V. A. Isaev, V. A. Koldanov, S. V. Kuzikov, J. L. Hirshfield, and S.H. Gold, Radiophys. Quantum Electron. 51, 597 (2008).

[5] A. L. Vikharev, A. M. Gorbachev, O. A. Ivanov, V. A. Isaev, S. V. Kuzikov, M. A. Lobaev, J. L. Hirshfield, S. H. Gold, and A. K. Kinkead, Phys. Rev. ST Accel. Beams 12, 062003 (2009).

[6] S. G. Tantawi, R. D. Ruth, A. E. Vlieks, and M. Zolotorev, IEEE Trans. Microwave Theory Tech. 45, 1486 (1997).

[7] F. Tamura and S. G. Tantawi, Phys. Rev. ST Accel. Beams 5, 062001 (2002).

[8] S. Yu. Kazakov, S. V. Shchelkunov, V.P. Yakovlev, A. Kanareykin, E. Nenasheva, and J. L. Hirshfield, Phys. Rev. ST Accel. Beams 13, 113501 (2010).

[9] A. L. Vikharev, A. M. Gorbachev, O. A. Ivanov, V. A. Isaev, S. V. Kuzikov, and M. A. Lobaev, Tech. Phys. Lett. 33, 785 (2007).

[10] A.B. Pippard, The Physics of Vibration (Cambridge University Press, New York, 1989). 
[11] V.E. Golant, Microwave Methods of Plasma Diagnostics (Nauka, Moscow, 1968).

[12] A. L. Vikharev, A. A. Vikharev, A. M. Gorbachev, O. A. Ivanov, V. A. Isaev, S. V. Kuzikov, and M. A. Lobaev, Tech. Phys. 54, 1648 (2009).
[13] O. A. Ivanov, V. A. Isaev, M. A. Lobaev, A. L. Vikharev, and J.L. Hirshfield, Appl. Phys. Lett. 97, 031501 (2010).

[14] A. V. Gunin et al., IEEE Trans. Plasma Sci. 28, 537 (2000). 